\title{
Advances in detonation driving techniques for a shock tube/tunnel
}

\author{
H. R. Yu • H. Chen $\cdot$ W. Zhao
}

Received: 20 December 2004 / Accepted: 6 June 2005 / Published online: 22 July 2006

(C) Springer-Verlag 2006

\begin{abstract}
Early works on the detonation driven shock tube are reviewed briefly. High initial pressure detonable mixture can be used in backward-detonation driver when the buffer tube is attached to the end of the driver for eliminating the excessive reflected peak pressure. Experimental data showed that an improvement on attenuation of the incident shock wave generated by the forward driver can be obtained, provided the diameter of the driver is larger than that of the driven section and an abrupt reduction of cross-section area is placed just beyond the diaphragm. Also, it is clearly verified by a numerical analysis. An additional backward-detonation driver is proposed to attach to the primary detonation driver and on condition that the ratios of initial pressure in the additional driver to that in the primary driver exceed the threshold value, the Taylor wave behind detonation wave in the primary detonation driver can be eliminated completely.
\end{abstract}

Keywords Shock tube $\cdot$ Shock tunnel $\cdot$ Gaseous detonation

PACS 47.40.-x $\cdot$ 47.40.Rs $\cdot 47.40 . \mathrm{Nm}$

Communicated by K. Takayama.

H. R. Yu $(\bowtie) \cdot$ H. Chen · W. Zhao

Laboratory of High Temperature Gas Dynamics,

Institute of Mechanics, Chinese Academy of Sciences,

Beijing 100080, China

e-mail: yuhr@imech.ac.cn

\section{Introduction}

In view of the enthalpy and pressure requirements for hypervelocity ground test, the shock tunnel must incorporate a high performance driver. Among the existing driving techniques, only a few of them are qualified for the high performance driver. The detonation drivers are capable of producing high enthalpy and high pressure test flow simultaneously besides a easy operation and low capital investment. A brief summary of early works will be presented before the description of the recent developments on detonation drivers.

In the experiment on combustion driver, Hertzberg and Smith [1] unexpectedly found that the measured strength of incident shock wave exceeded the calculation on the assumption of normal shock tube flow and the constant volume combustion. In an endeavour to explaining this, they postulated a form of constant-pressure combustion, in which the burning gases issue from the high pressure end at constant pressure and the shock strength is such that the pressure ratio across it is equal to the original pressure ratio across the diaphragm. However, Bird [2] considers this picture is incompatible with any of the physically possible combustion processes in gases.Gerard (quoted from Bird [2]) attributed the phenomenon to a detonation which was happening in the driver. Subsequently, Bird [2] gave the verification of Gerard's judgment by the analysis of wave processes in the detonation driver and combustion driver.

In the view of practical purpose, two sites of detonation initiation are feasible, namely the forward and backward detonation driver modes. In the forward-detonation driver, the detonation wave is initiated at the closed end of the driver, and it moves downstream as 
same direction as the incident shock wave propagation. In the backward-detonation driver, the detonation wave is initiated at the main diaphragm, and it runs upstream with opposite direction to the incident shock wave propagation.

Waldron [3] carried out the experiments to investigate the driving behavior of forward-detonation driver. The driver gas is produced by a detonation initiated at the closed end of the driver, a manifest increase in shock velocity could be obtained for the same initial condition as when a constant volume burning is used. This is agreed with Bird's prediction. However, the shock attenuation is serious, it does not seems likely that a detonation can be used to produce the driver gas for generating a strong shock waves except the attenuation of the shock is unimportant. Balcerzak and Johnson [4] applied the forward-detonation driver to simulate a blast wave.

$\mathrm{Yu}$ [5] performed the experiments on backward-detonation driver. The results indicated that the strength of shock wave generated by backward-detonation driver is stronger than that by the combustion driver under otherwise identical conditions. It is different from Bird's prediction because Bird did not consider the effect of wall heat loss on combustion or detonation. As the propagation speed of flame is much slower than that of detonation wave, the heat loss of the former is more serious than the latter. The attenuation of the incident shock wave generated by backward-detonation driver is quite weak and the experimental results show a good repeatability. Nevertheless, the reflection of the detonation produces considerable overpressure on the end of the tube so that the allowable initial pressure is restricted within a low pressure scope. Accordingly, the backward-detonation driver was not available for the practical hypervelocity ground test facilities at that time.

In Sect.2, the method for eliminating the reflected pressure at the end of backward-detonation driver is discussed. Section 3 makes a behavior comparison between backward-detonation driver and heated hydrogen driver. Section 4 presents methods for alleviating detrimental effect of Taylor rarefaction wave. In Sect. 5, the double detonation drivers for eliminating Taylor rarefaction are shown.

\section{Method for eliminating the reflected pressure at the end of backward-detonation driver}

In the backward-detonation driver, the detonation wave is initialed near the main diaphragm and propagates upstream to the closed end of the driver. When the detonation reaches the end plate, the high pressure over hundred times of the initial pressure arises from the reflection of the detonation [6].

Yu et al. $[7,8]$ proposed to attach a buffer tube to the closed end of the driver tube for eliminating the excessive reflected pressure. In Fig. 1, the sketch and the wave diagram of flow in backward-detonation driven shock tube with a buffer tube are plotted. The light diaphragm between the buffer and detonation tube will be ruptured immediately when the detonation front reaches. As the initial pressure of buffer tube is much lower in comparison with that of the detonation driver, the reflected wave will be rarefaction waves instead of a shock wave. By this way, the high reflected pressure disappears at the closed end of the backward-detonation driver, and it makes high initial pressure of detonable mixture available for a practical application in the shock tube driving.

The flow process in the buffer tube is equivalent to that in the driven section of forward-detonation detonation driven shock tube which consists of detonation driver and buffer tube. According to the theory of shock tube flow, it is well known that the pressure behind a reflected shock wave is steadily decreasing along with the drop of the initial pressure in the driven section without changing the other initial parameters. The experimental relationships between the reflected pressure peak and the initial pressure in the buffer tube are shown in Fig. 2 [9], in which two kinds of buffer gases were tested and the reflected pressure peak drops exponentially when the buffer is nearly vacuumed. Accordingly, the lower the initial pressure in the buffer tube is, the weaker the shock load on the end of buffer tube.

The second advantage of the buffer tube lies in the increase of the duration of constant driver properties. Without a buffer tube the detonation wave reflects at



Fig. 1 Wave diagram of flow in detonation driven shock tube with a buffer tube 


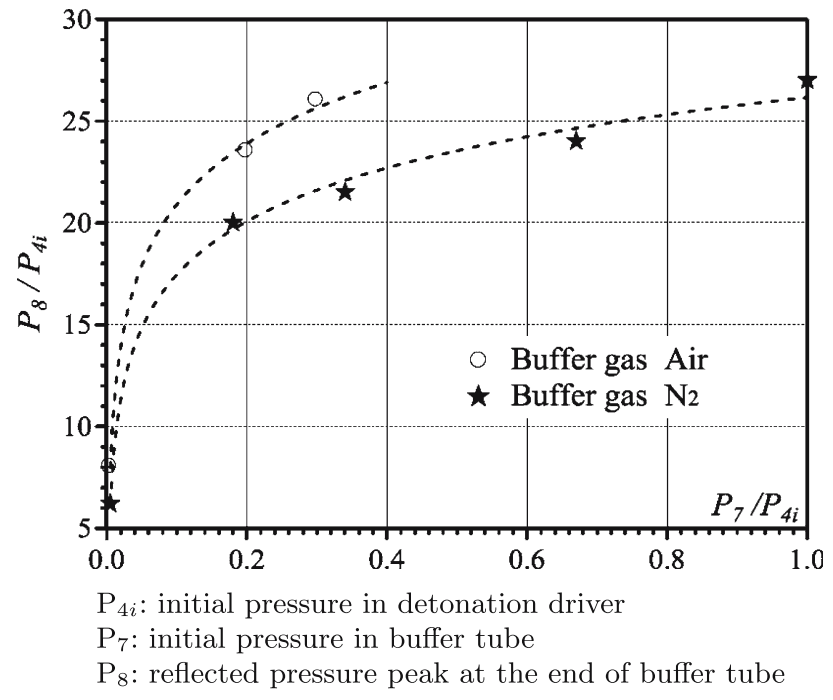

Fig. 2 Reflected peak pressure versus initial pressure in buffer tube

the end wall and then propagates downstream as a shock wave. In case a buffer tube is attached to the driver, a reflected rarefaction wave is generated at the diaphragm station between the driver and buffer tube. Because the head of the reflected rarefaction wave travels slower than the shock, the buffer tube yields a longer flow duration of constant driver condition.

\section{Driving quality of back-detonation driver}

Presently, backward-detonation driven shock tubes/tunnels were constructed in the university of Texas, Arlington; the RWTH Aachen and the Institute of Mechanics, CAS, Beijing [10].

Experimental observations on gaseous detonation have shown that the real structure is much complex. However, for the purpose of detonation driving techniques, the ideal ZND wave structure is good average representation. A detonation which is initiated at the main diaphragm running backwards to the end wall of the driver, a constant state (region 4 in Fig 1) behind the Taylor rarefaction wave followed detonation wave develops [11]. The gases in region 4 are analogous to driver gas in normal shock tube, therefor a backward detonation driver is qualified for high grade.

The experimental results on the attenuation of incident shock wave are illustrated in Fig. 3, including the data of both backward-detonation driven shock tube [12] and heating hydrogen driven shock tube [13]. A comparison can be made from the performance of these two driver section, Since their driven section have the identical inner diameter $(100 \mathrm{~mm})$ and similar initial

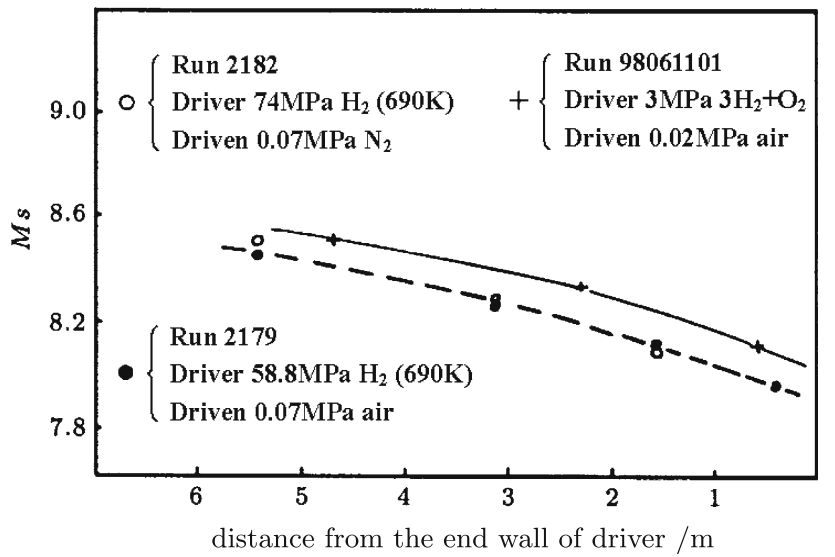

Fig. 3 Attenuation characteristics of the incident shock waves

pressure and length. From Fig. 3 it can be seen that the driving quality of backward-detonation driver corresponds to that of heating hydrogen driver.

\section{Methods for alleviating detrimental effect of Taylor wave}

The driving capability of forward-detonation driver is much stronger than that of backward-detonation driver. However, the Taylor rarefaction fan behind detonation wave will continuously catch up with the incident shock wave and weaken the shock strength in the forward-detonation driven shock tube. If the detrimental effect of the Taylor wave can be alleviated to a extent of practical feasibility, the forward-detonation driver may be the better choice for hypervelocity ground test facilities.

Several conceptions were proposed to reduced the detrimental influence of Taylor wave.

\subsection{Lengthening the driver}

The flow field produced by detonation wave in a closed tube consists of a constant-velocity detonation itself followed by a self-similar Taylor wave that brings the post-detonation gas set in motion back to rest [14]. The length of the drop region including pressure, temperature and velocity is about one-half of the distance which the detonation wave front has propagated. The longer the distance, the smaller the drop per unit length for the pressure, temperature and velocity because the total amount drops are fixed. It means that a longer detonation driver can provide a relative uniform driving flow behind the detonation wave. However, this method is limited due to lower efficiency. 


\subsection{Increasing diameter of the driver}

The Mach numbers of incident shock wave can be increased by utilizing a driven section with a reduced in cross-section area [15]. In the case of using a detonation, when the detonation wave initiated at the closed end reaches the convergent section, there will form a reflected shock wave which will interact with the opposing rarefaction wave, see Fig. 4; the rarefaction fan will be expanded; then the decay of state parameters in driving flow is alleviated. Meanwhile, the gas in the outer ring continuously fills into the main flow from lateral side, which makes further the flow field uniform. Therefore, the attenuation of incident shock wave can be weakened substantially, which was demonstrated by the experimental results [12].

The numerical analysis about the behavior of a variable cross-section forward-detonation driven shock tube was studied by Yang [16], whose results (Fig. 5) show that the half conic angles of converging section dominate the attenuation of incident shock wave along the driven section. Although the maximum Mach number of incident shock wave generated by a variable cross-section shock tube is identical with the one by an uniform detonation tube, the former shock attenuation is much weaker than the latter when the half conic angle of converging section is about $90^{\circ}$. Within the range of half conic angle $\beta \leq 75^{\circ}$, the maximum Mach number and the shock attenuations are steadily raising along with the decrease of the half conic $\beta$ angle. Hence the optimum half conic angle $\beta$ to alleviate the detrimental effect of Taylor wave should be $90^{\circ}$.

The experimental results [12] demonstrates that the attenuation of incident shock wave was alleviated to be

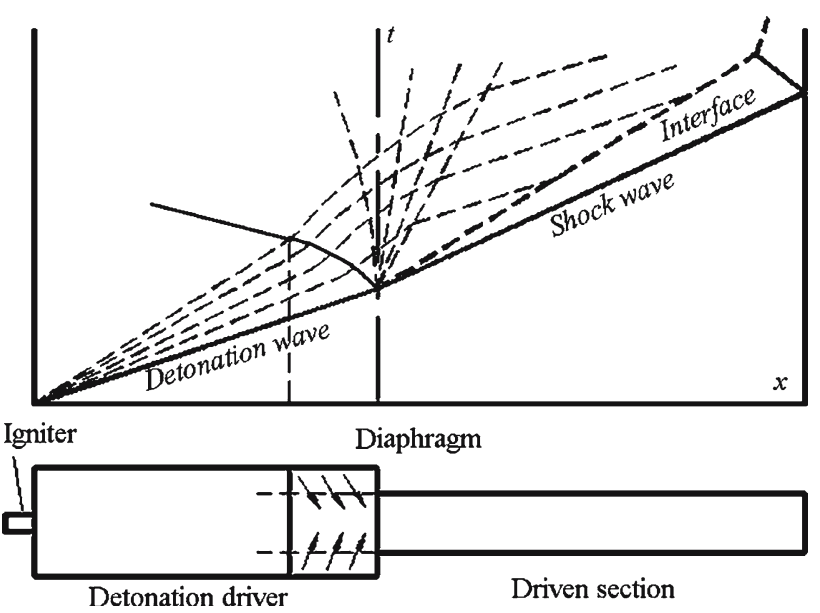

Fig. 4 Diagram of flow in variable cross-section shock tube with forward detonation driver

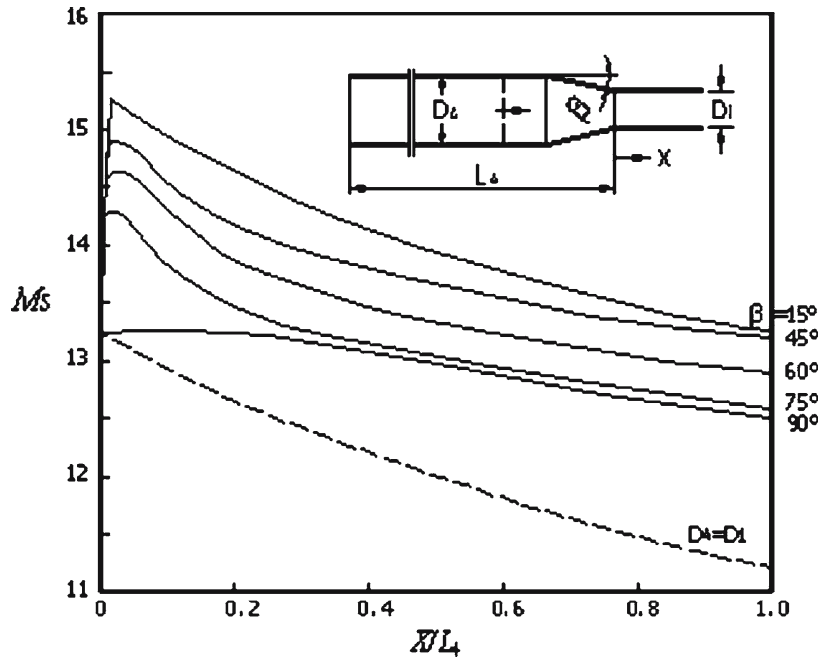

Fig. 5 Variations of Mach number of incident shock wave

acceptable by increasing length and diameter of forward detonation driver.

\subsection{Inserting a cavity ring}

Effects of variable cross-section area of detonation tube on the uniformity of flows followed the detonation wave were studied numerically by Jiang et al. [17]. It is found that the flow field behind a detonation wave generated by the tube with a cavity ring is more uniform than that by the tube with a $30^{\circ}$ or $45^{\circ}$ converging section. In order to verify the results, a forward-detonation driven shock tube has been manufactured in LHD [18]. The detonation driver consists of a large tube $(3.87 \mathrm{~m}$ long and $90 \mathrm{~mm}$ in Dia. ), a cavity ring (130 $\mathrm{mm}$ in Dia. ) and a small tube $(180 \mathrm{~mm}$ long and $60 \mathrm{~mm}$ in Dia. $)$ in the order. The driven section is $7 \mathrm{~m}$ long and $60 \mathrm{~mm}$ in Dia. . According to the measured pressure histories, the driver with the cavity ring ( $360 \mathrm{~mm}$ long) can generate uniform stagnation gas.

\section{Method of eliminating the Taylor wave}

Only if the Taylor rarefaction fan which follows the detonation wave is eliminated completely, the performance of forward-detonation driver can be improved thoroughly.

If a moving piston is following a detonation wave and its velocity reaches or exceeds $C-J$ value of the detonation product, the Taylor wave will disappear. Moreover, what follows the detonation wave will be a gas column with constant velocity and uniform state parameters. Because the velocity of detonation product is very 
high, it is difficult to accelerate a mechanical piston to such high speed in a shock tube. Coats and Gaydon [19] employed first an additional driver utilizing hydrogen as the driving gas attached to the upstream end of the detonation driver, which generated a moving gaseous piston instead of a traveling mechanical piston. Bakos et al. [20] took helium as driving gas in the additional driver. They employed the pressure of unheated hydrogen or helium are all rather lower, therefore the Taylor wave can not be eliminated completely and the initial strength of the shock in detonable mixture is not strong enough to direct initiate the detonation wave reliably.

For equaling the velocity and pressure of the gaseous piston to that of the detonation product respectively, the initial pressure unheated hydrogen or helium should exceed 100 times higher than that of detonable mixture in the detonation section. The initial pressure of detonable mixture in the driver of practical shock tunnel is most often several MPa or more. Therefore, the required initial pressure of hydrogen or helium must be excessively high in order to eliminate the Taylor wave completely. Not only are the required high-pressure pump and vessel equipment too expensive, but also the structures of the additional driver as well as the rupture technology of thick diaphragm present severe technical problem.

In order to avoid these technical problems, a backward-detonation driver utilizing the same detonable mixture in the primary detonation driver is employed as the additional driver instead of the light gas drivers. Under these conditions, the initial pressure of the additional driver need only be several times higher than that of the primary driver for eliminating the Taylor wave completely. In addition, the post-detonation gas ejected from the additional driver can directly initiate a detonation wave in the primary driver. Thus, another technical impediment is also solved in practice.

\subsection{Estimation of the critical ratio of initial pressure}

The double detonation driven shock tube, shown schematically in Fig. 6, consists of both the additional backward-detonation driver and the primary forwarddetonation driver in which the same oxy-hydrogen mixture are utilized and a driven section. The post-detonation gases in the additional driver penetrating the Taylor wave are decelerated to the stationary state and then accelerated to the $\mathrm{u}_{6}$ after penetrating the central rarefaction wave caused by the rupture of the diaphragm. According to the relation of simple wave :

$$
u_{C J}+\frac{2}{(\gamma-1)} a_{C J}=u_{6}+\frac{2}{(\gamma-1)} a_{6}
$$

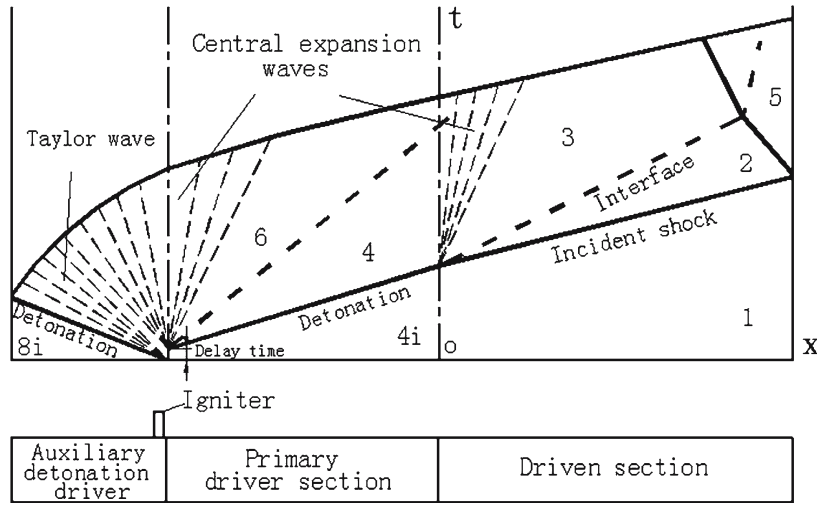

Fig. 6 Wave diagram of flow in a shock tube with double detonation drivers

On the assumption that (1) the ratio of the hydrogen to oxygen is 3 ; (2) the initial temperature of oxyhydrogen mixtures is $293^{\circ} \mathrm{K}$; (3) the initial pressure of the mixtures does not effect the ratios of pressure and sound-speed of the detonation wave front to the wave rear; (4) the special heat ratio of post-detonation gases in the expansion process is constant $(\gamma=1.2)$; (5) the Taylor wave following the primary detonation is just eliminated completely. We can obtained:

$u_{6}=u_{4}=u_{C J}$

$a_{6}=a_{C J}-(\gamma-1) u_{C J}$

Based on the values quoted from Edwards et al. [6], $u_{C J}=1.4 \times 10^{3} \mathrm{~m} / \mathrm{s}, a_{C J}=1.8 \times 10^{3} \mathrm{~m} / \mathrm{s}$,

Then $a_{6}$ is deduced as:

$a_{6}=1.52 \times 10^{3} \mathrm{~m} / \mathrm{s}$

The critical initial pressure ratio between the additional and the primary detonation driver is

$\frac{P_{8 i}}{P_{4 i}}=\frac{\left(P_{C J}\right)_{a}}{\left(P_{C J}\right)_{p}}=\left(\frac{a_{C J}}{a_{6}}\right)^{\frac{2 \gamma}{\gamma-1}}=7.6$

The corresponding critical initial pressure ratios for the unheated light gas are about 110 times $\left(\mathrm{H}_{2}\right)$ and 540 times $(\mathrm{He})$, respectively.

An overdriven detonation wave will be generated in the primary driver if the ratio of the initial pressure exceeds the critical value. Meanwhile, the velocity, sound-speed and pressure of the detonation product are increased also. Therefor, the driving capability is steadily strengthened with the increasing of the ratio of the initial pressure.

\subsection{Experimental results}

The additional and primary driver are filled with the same detonable mixture of $3 \mathrm{H}_{2}+\mathrm{O}_{2}$ for all experiments. 
Three measured pressure histories at the primary detonation driver under different driving conditions are dipicted in Fig. 7 [21]. Figure 7a is measured under a single forward-detonation driver mode, in which the pressure jumps instantaneously to be almost 18 times higher than the initial value due to the arrival of detonation wave, then it gradually dropped below half of the peak value because of the Taylor wave, at last it keeps a constant value. Figure $7 b$ and $c$ are measured under the double detonation driver mode. Although the pressure jumps abruptly and then dropped also in Fig. 7b, the duration of the drop was shorter and the pressure platform value is higher than Fig. 7a obviously. In the case of Fig. 7b, only part of the Taylor wave is eliminated because the initial pressure ratio $\left(\mathrm{P}_{8 i} / \mathrm{P}_{4 i}=4\right)$ was lower than the critical value. Because the initial pressure ratio is close to the critical value in Fig. $7 \mathrm{c}\left(P_{8 i} / \mathrm{P}_{4 i}=6\right)$, the pressure does not drop after it jumped up at the beginning, which means the Taylor wave was almost eliminated. Both in Fig. $7 \mathrm{~b}$ and $\mathrm{c}$, the second pressure jumps were caused by the overtaken of the reflected shock waves from the end of the additional driver. Such interferences can be eliminated if the length of each section is designed properly.

Under the condition of the same of initial pressures and of the maximal Mach numbers in the driver section, the comparison of the Mach number variations of the incident shock wave generated by double detonation drivers with those caused by single forward detonation driver are shown in Fig. 8. It shows that the attenuation of an incident shock wave generated in the double drivers is weaker than that by a single detonation driver. However, the attenuation of incident shock was appreciable, even for a wave generated in the double detonation driver because the attenuation was dependent not only the quality of the driver gas, but also on boundary layer of the driver section wall. For the apparatus used in this work, the surface state of the tube wall was not ideal and the initial pressure in driven section was lower, inevitably boundary layer effect induced substantial attenuation of the incident shock wave. In practical applications, we can improve the state of the tube wall and increase the initial pressure, thus the boundary layer effect will decrease considerably and the attenuation of the incident shock wave generated by the double detonation driver will be reduced significantly.

\section{Conclusions}

The driving capability of a forward-detonation driver is more powerful than that of a backward-detonation driver. Only either the rarefaction fan following detonation wave is eliminated completely or its detrimental

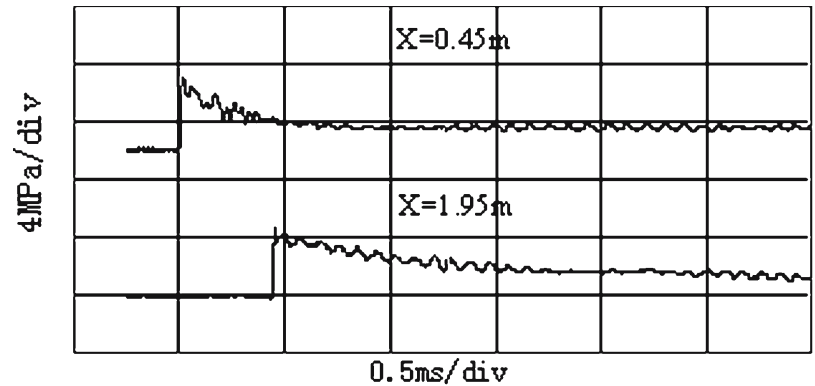

(a) Single detonation driver $\left(\mathrm{P}_{4 \mathrm{i}}=0.25 \mathrm{MP}\right.$ a $)$

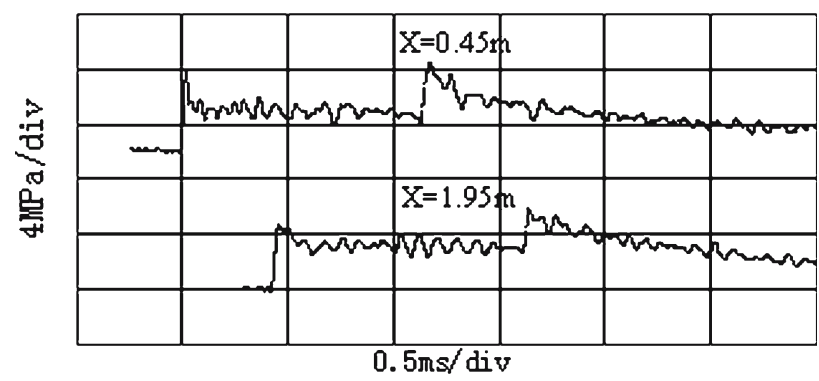

(b) Double detonation drivers $\left(\mathrm{P}_{8} / \mathrm{P}_{4 i}=4, \mathrm{P}_{4}=0.25 \mathrm{MP}\right.$ a)

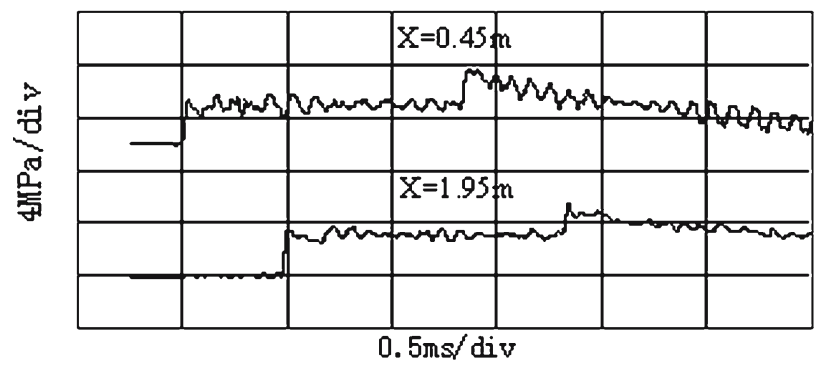

(c) Double detonation drivers $\left(\mathrm{P}_{8 \mathrm{ij}} \mathrm{P}_{4 \mathrm{i}}=6, \mathrm{P}_{4 \mathrm{i}}=0.2 \mathrm{MP} \mathrm{a}\right)$

Fig. 7 Pressure histories in the primary driver



Fig. 8 Attenuation characteristics of incident shock wave along then shock tube

influence is alleviated to an acceptable extent for practical application, the forward-detonation driver can be available to the aerodynamic ground test facilities. Both the additional and the primary detonation drivers filled with the same oxyhydrogen mixture is proposed to eliminate the Taylor wave in the primary detonation driver. 
The distribution of the pressure, temperature and velocity behind the detonation wave front can be eliminate along the primary driver section. Namely the Taylor wave is eliminated completely when the ratio of initial pressure in the additional driver to that in the primary driver equals or exceeds the threshold value. On condition that initial pressure ratios exceed the threshold values, the state parameters and the velocity of postdetonation gas in the primary detonation driver will be steadily increased with the ratio of initial pressure heightening. Consequently, it offers a new way to further raise the enthalpy of the test flow.

Acknowledgements We express our cordial thanks to Prof. Grönig and Dr. F. Zhang who kindly supported us to start preliminary experiment for improving performance of detonation drivers in Aachen Shock Wave Laboratory. Also, thanks go to a number of students and colleagues of the Key Laboratory of High Temperature Gas Dynamics, CAS, especially, H. Feng and J.M. Lin. This work was supported by National Foundation of Nature Science of China (10082004).

\section{References}

1. Hertzberg, A., Smith, W.E.: A method for generation strong shock waves. J. Appl. Phys. 25, 130 (1954)

2. Bird, G.A.: A note on combustion driven shock tubes, RAE TN-Aero 2511, R.A.E. Farnborough, England (1957)

3. Waldron, H.F.: An experimental investigation of the flow properties behind strong shock wases in nitrogen, UTIA Report, No.50 (1958)

4. Balcargak, M., Johnson, M.R.: The gaseous-detonation driver and its application to shock tube simulation technigues.In: Proceedings of 5th International Shock Tube Symposium, pp.1111-1128, White Oak, Maryland (1996)

5. Yu, H.R.: Shock tunnel and its application to aeroheating experiments, Thesis, Institute of Mechanics, Chinese Academy of Sciences (In Chinese) (1963)

6. Edwards, D.H., Williams, G.T., Breege, J.C.: Pressure and velocity measurements on detonation waves in hydro-oxygen mixtures. J. Fluid Mech. 6, 497-517 (1959)
7. Yu, H.R.: Recent developments in shock tube application. In: Takayama, K. (ed.) Proceedings of 1989 National Symposium on Shock Wave Phenomena, Sendai, Tohoku University (1989)

8. Yu, H.R., Esser, B., Lenartz, M., Groenig, H.: Gaseous detonation driver for a shock tunnel. Shock Waves 2(4), 245-254 (1992)

9. Yu, H.R., Zhao, W.: The use of oxy-hydrogen detonation driver for generator of high enthalpy flow. In: Shen, C. (ed.) Proceeding 20th International Symposium on Rarefied Gasdynamic, pp. 927-933 (1997)

10. Lu, F.K., Marren, D.E.: Advanced hypersonic test facilities. Progress in Astronautics and Aeronautics, vol.198, AIAA, Virginia, pp. 137 (2002)

11. Nettleton, M.A.: Gaseous detonation. Chapman and Hall,London (1987)

12. Yu, H.R.: Oxy-hydrogen combustion and detonation driven shock tube. Acta Mech. Sin. 15(2), 97-107 (1999)

13. Fuehrer, R.G.: Measurements of incident shock test time and reflected shock pressure at full turbulent boundary layer test conditions. In: Glass, II. (ed.) Shock Tubes. University Toronto press, pp. 31-59 (1970)

14. Taylor, G.I.: The dynamics of the combustion products behind planar and spherical detonation fronts in explosive. Proc. R. Soc. (London) A 200, 235-247 (1950)

15. Alpher, R.A., White, D.R.: Flow in shock tubes with area change at diaphragm section. J. Fluid. Mech. 8:457 (1958)

16. Yang, H.W., Huang, D., Yu, H.R.: Numerical simulation of the behavior of a variable cross-section forward-detonation driven shock tube, Acta Mechanica Sinica (In Chinese), 37(4), 491-500 (2005)

17. Jiang, Z.L., Yu, H.R., Takayama, K.: Investigation into converging gaseous detonation driver. In: Proceedings of 22nd ISSW, London(1999)

18. Jiang, Z.L., Zhao, W., Wang, C., Takayama, K.: Forwardrunning detonation driver for high-enthalpy shock tunnels. AIAA J. 40(10), 2009-2016 (2002)

19. Coates, D.H., Gaydon, F.R.S.: A simple tube with detonating driver gas. Proc. R. Soc (London) A 283, 18-32 (1965)

20. Bakos, R.J., Calleja, J.F., Erdos, J.I.: An experimental and computational study leading to new test capabilities for the HYPULSE facility with a detonation driver. AIAA 96-2193 (1996)

21. Chen, H., Feng, H., Yu, H.R.: Double detonation drivers for a shock tube/tunnel, Science in China. Ser. G 47(4), 502-512 (2004) 